\title{
Introduction to the special issue of papers from DISC 2015
}

\author{
Yoram Moses ${ }^{1}$
}

Published online: 25 June 2018

(c) Springer-Verlag GmbH Germany, part of Springer Nature 2018

This special issue of Distributed Computing is based on four papers that originally appeared, in preliminary and abbreviated form, in the Proceedings of the 29th International Symposium on Distributed Computing (DISC), held on October 7-9, 2015 in Tokyo, Japan. The papers were chosen by the Program Committee from the 42 full-length papers presented at the symposium, based on their quality and representation of the range of topics addressed in the symposium.

DISC is an annual forum for presentation of high-quality and novel research on all aspects of Distributed Computing, including the theory, design, implementation and applications of distributed algorithms, systems and networks. The four selected papers offer a fascinating view of the breadth and depth of subjects presented at DISC.

The first paper, by Rati Gelashvili, introduces a new technique for proving space lower bounds for the fundamental problem of consensus in shared memory systems, which results in new insights and greatly improved bounds.

The second paper, by Michael Dinitz, Jeremy Fineman, Seth Gilbert and Calvin Newport, shows how the smoothed analysis technique originally introduced by Spielman and Teng for the analysis of sequential algorithms can be adapted to distributed protocols and can provide new insights into their behavior.
The third paper, by David Doty and David Soloveichik proves new bounds on the parallel time a population protocol needs to elect a leader. Its results are valid in chemical reaction networks, and provide insights into systems of biological cells.

Finally, the fourth paper, by Eric Goubault, Samuel Mimram and Christine Tasson, expands the mathematical tools available for the study of distributed problems by relating solvability and computability in distributed systems to directed algebraic topology.

In addition to being reviewed, in preliminary form, by the program committee of DISC 2015, the papers selected for this special issue were subsequently handled by the journal's editors and referees according to the standard practices of Distributed Computing. I wish to thank the authors, the referees and the journal's handling editors for their contributions to this issue.
Yoram Moses

moses@ee.technion.ac.il

1 Department of Electrical Engineering, Technion, Haifa, Israel 